\title{
Perceived locus of control, expectancy, and choice reaction time
}

\author{
E. SCOTT GELLER \\ Virginia Polytechnic Institute and State University, Blacksburg, Virginia
}

\begin{abstract}
Prior to each of 200 stimulus presentations in a two-choice reaction time (RT) paradigm, subjects made a stimulus prediction and then estimated their prediction confidence by verbalizing a number from 50 to 100 . The probability of a correct stimulus prediction was experimenter controlled at .70 for a competent condition and at .30 for an incompetent condition. Half of the subjects in each condition were internals $(\mathrm{N}=12)$ and half were externals, as defined by Rotter's Locus of Control Scale. An external locus of control and an incompetent task competency were additive in increasing independence among three expectancy indexes: stimulus prediction, prediction confidence, and choice RT. Specifically, for prediction-competent internals, the dependency among prediction outcome, confidence, and RT was exactly as hypothesized from expectancy theory; that is, RT was markedly faster to correctly predicted than to incorrectly predicted stimuli, RT to correctly predicted stimuli decreased as confidence in the prediction increased, and RT to incorrectly predicted stimuli increased as confidence for the predicted (nonpresented) stimulus increased. In contrast, neither prediction outcome nor prediction confidence significantly influenced the RT of prediction-incompetent externals.
\end{abstract}

Expectancy is a mediating construct, widely used to interpret environment-behavior relationships. For example, personality theorists and researchers define the perceived-locus-of-control dimension (Rotter, 1966) by referring to one's general expectancy that individual circumstances are affected by personal action, whereas investigators of human information processing emphasize expectancy-determining task characteristics when interpreting differential performances. To study particular hypotheses generated from Rotter's (1966) locus-of-control concept, the present experiment applied a sensitive behavioral indicator of expectancy that has been used often by investigators of cognitive processing (i.e., choice reaction time, or choice RT). In particular, the choice RTs of individuals scoring as internals and externals on Rotter's (1966) Perceived Locus of Control Scale were compared, on the assumption that such RTs reflect expectancies mediating decisions and consequences.

When subjects were required to predict verbally the trial-by-trial stimulus occurrences in a choice RT task, choice RT was markedly faster to correctly predicted than to incorrectly predicted stimuli (e.g., Geller, 1974; Geller \& Whitman, 1973). Furthermore, when subjects indicated their confidence in each stimulus prediction by verbalizing a probability from 50 to 100 , choice RT varied according to an expectancy interpre-

This research was supported in part by Grant MH27219-02 from the National Institute of Mental Health. The author is grateful for the research assistance of Mark Albert, Mark Lewis, and Charles Whitman. Requests for reprints should be sent to E. Scott Geller, Department of Psychology, Virginia Polytechnic Institute and State University, Blacksburg, Virginia 24061. tation. That is, choice RT not only was faster to stimuli that had been predicted (rather than nonpredicted), but RT to correctly predicted stimuli also decreased linearly as the confidence judgment associated with the stimulus prediction increased (Geller \& Whitman, 1973). In the present study, the relationship between choice RT and prediction confidence was compared for internals and externals. Given the theory that internals are more apt to perceive a causal relationship between personal behavior and consequence (see Lefcourt, 1976), it was hypothesized that choice RT (an indicator of one's expectancy mediating the prediction and occurrence of a stimulus) would be more influenced by the predictions and confidence judgments of internals than by those of externals.

Geller (1974) and Whitman and Geller (1973) found choice RT to vary significantly as a function of subjects' relative success at correctly predicting stimulus presentations. The relationship between prediction success and choice RT was similar to that between prediction confidence and choice RT; that is, choice RT to correctly predicted events decreased linearly as the probability of a correct prediction increased. In the present study, the prediction success of internals and externals was controlled at levels of $70 \%$ correct or $30 \%$ correct. Since internals are more likely than externals to attribute environmental contingencies to personal competencies, it is reasonable to hypothesize that internals will be more attentive and thus more responsive to task contingencies (see Lefcourt, 1976, and Phares, 1976). If this is the case, the choice RT of internals should be more influenced by relative prediction success. Furthermore, an independence between choice RT and verbal expectancy (indicated by stimulus predictions and pre- 
diction confidence) would reflect a release (or giving up) of perceived personal control, and such a phenomenon has been presumed to be more probable for externals than for internals (e.g., Hiroto, 1974; Lefcourt, 1976; Phares, 1976) and was actually found to be the case in two demonstrations of human learned helplessness (Albert \& Geller, 1978).

\section{METHOD}

\section{Subjects}

Students from introductory psychology classes at Virginia Polytechnic Institute and State University were given the Rotter (1966) Locus of Control Scale $(N=302)$. Students with a score of at least one standard deviation above or below the mean for their sex group (mean $=10.21$ for males and 11.59 for females) were assigned randomly to two experimental conditions, with the restriction that each condition include six males and six females who had scored above the mean (i.e., externals) and six males and six females who had scored below the mean (i.e., internals).

\section{Procedure}

Each subject was tested individually in 1-h sessions. The experimenter was unaware of the subject's score on the internalexternal scale. Each of the 210 trials of the experimental session (including 10 practice trials) began with the presentation of a horizontal line in the center of a stimulus readout, which signaled the subjects to predict which of two stimulus alternatives they expected to occur. The stimulus prediction was made by first verbalizing either "up" to predict the symbol $\sqcup$ or "down" to predict the alternative symbol $\Pi$, and then, with the left index finger, depressing one of two spring-return levers that were positioned on a panel located $40 \mathrm{~cm}$ to the left of a stimulus readout.

After making a stimulus prediction, the subjects estimated their degree of confidence in their prediction by verbalizing one of six numbers printed on a card above the stimulus readout: $50,60,70,80,90$, or 100 . The instructions stated that a confidence estimate of 50 should represent complete uncertainty in the prediction, and that 100 should indicate complete certainty. Furthermore, the instructions identified a skill aspect of the prediction task by suggesting that the stimuli would intermittently show sequential patterning, and that the subject's prediction score (i.e., the number of correct stimulus predictions) would depend partially upon his/her ability to decipher and anticipate regularities in the stimulus series.

Following the subject's prediction and confidence statement, a .3-sec "ready" buzzer sounded; then, after a random time interval ranging from .5 to $1.5 \mathrm{sec}$, the symbol $\sqcup$ or $\Pi$ was presented by the illumination of a $1.5 \times 2.5 \mathrm{~cm}$ digital readout, centered on a black partition separating the experimenter from the subject. The subject identified the stimulus presentation as quickly as possible by pulling a right-hand or left-hand reaction trigger. The identification response turned off the stimulus and stopped a digital millisecond timer that had started upon stimulus presentation.

Relay logic equipment combined the result of subjects' selections of a prediction switch (corresponding to their verbal prediction) with information from a paper-tape reader in order to determine whether the predicted or the nonpredicted stimulus would occur. The probability of a correct prediction was controlled at .70 for one half of the subjects (12 internals and 12 externals) and at .30 for the remaining 12 internals and 12 externals. The subjects were told that the stimulus sequence was predetermined on a punched paper tape (even though the tape actually controlled prediction success).

\section{RESULTS}

The trials on which the subjects made anticipatory or incorrect identifications were deleted from the RT analysis; these trials did not exceed 5\% (i.e., 10) for any session. Furthermore, error frequency did not vary reliably as a function of the between-subject conditions: locus of control and prediction success. For each subject, the response latencies to make correct stimulus identifications were dichotomized according to the outcome of the subject's prediction (correct or incorrect) and then were classified according to the subject's confidence in the prediction. Choice RTs on trials on which the prediction confidence estimate had been 50,60 , or 70 were included in one category, and RTs on trials on which the confidence had been 80,90 , or 100 were included in a second category. The category means were computed for each subject, and then group averages were calculated. Consequently, the analysis of variance (ANOVA) included two between-subject and two within-subject variables: 2 (locus of control-internals vs. externals) x 2 (prediction success-70\% correct vs. $30 \%$ correct) $\times 2$ (prediction outcome-correct vs. incorrect) $\times 2$ (confidence categories-50, 60, 70 vs. $80,90,100)$.

Significant main effects of prediction outcome and confidence were obtained $[\mathrm{F}(1,44)=46.55, \mathrm{p}<.001$, and $F(1,44)=5.79, p<.025$, respectively] . The following three interactions reached significance at the .05 level: prediction outcome $x$ prediction success $[F(1,44)$ $=8.35, \mathrm{p}<.01]$, prediction outcome $\mathrm{x}$ confidence $[F(1,44)=4.23, p<.05]$, and locus of control $x$ prediction success $x$ confidence $[F(1,44)=4.33, p<.05]$.

To study the nature of the interactions, a separate analysis was completed for each between-subject variable, with the error estimate provided by the overall ANOVA. That is, a 2 (prediction outcome) $\times 2$ (confidence)

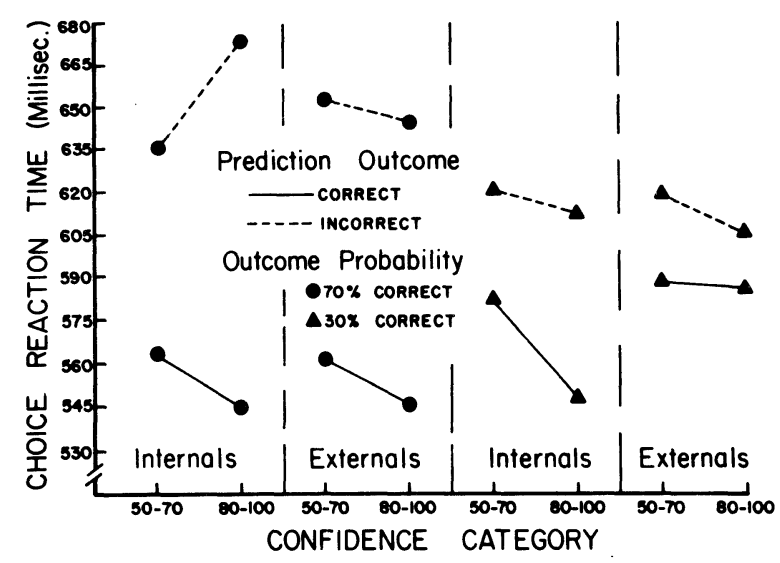

Figure 1. Choice RT as a function of two between-subject variables (perceived locus of control and prediction success) and two within-subject variables (prediction confidence and prediction outcome). 
ANOVA was completed for each of the four panels in Figure 1.

For internals on the $70 \%$-correct schedule (i.e., the leftmost panel of Figure 1), choice RTs to correctly predicted stimuli were an inverse function of the confidence category, but RTs to incorrectly predicted stimuli were a direct function of confidence. The analysis indicated a significant main effect of prediction outcome $[F(1,44)=23.06, p<.001]$ and a significant prediction outcome $x$ confidence interaction $[F(1,44)=7.08$, $\mathrm{p}<.025]$. For the two middle panels of Figure 1 (70\%-correct externals and 30\%-correct internals), significant main effects of both confidence outcome and prediction outcome were obtained (ps $<.025)$, and the interactions were not reliable (both ps $<.10$ ). The ANOVA for 30\%-correct externals (i.e., the rightmost panel of Figure 1) demonstrated no significant effects (all ps $<.10$ ).

\section{DISCUSSION}

A verbal stimulus prediction, confidence in a stimulus prediction, and reaction latency to identify a stimulus presentation reflect one's expectancy for a stimulus occurrence (see Geller, 1974, and Geller \& Whitman, 1973). The present study found the relationship between these expectancy indexes to depend upon both the task competence of the subject (i.e., prediction success) and the subject's perceived locus of control (i.e., internal or external). Furthermore, both prediction competence and perceived locus of control affected the confidence-RT function in directions hypothesized from social learning theory (Rotter, 1954). More specifically, the relationship between choice RT and prediction confidence was most indicative of contingent changes in stimulus expectancy when prediction success implied that the subjects should place a relatively high degree of confidence in their predictions (i.e., 70\% correct predictions) and when the subjects' scores on Rotter's (1966) internalexternal scale suggested that they were particularly attentive to the prediction-outcome contingencies (i.e., internal locus of control). Thus, for this condition, choice RT indicated that the subjects' expectancy (or preparedness) for the stimulus they predicted increased directly with the confidence associated with their prediction. That is, when the predicted stimulus occurred, RT was faster for the high than for the low confidence categories; but when the predicted stimulus did not occur, RT was slower for the high than for the low confidence categories, because increased expectation for the predicted but nonpresented stimulus resulted in reduced readiness for the presented (nonpredicted) stimulus. It is noteworthy that Geller and Whitman (1973) did not obtain this direct relationship between subjects' prediction confidence and RT to incorrectly predicted stimuli, which had been hypothesized from the authors' (Geller \& Whitman's) expectancy theory. With randomly selected subjects (i.e., subjects not chosen on the basis of a personality scale) and randomly determined prediction success (i.e., $45 \%$ to $55 \%$ correct predictions per subject), Geller and Whitman found no effects of prediction confidence on choice RT to incorrectly predicted stimuli; that is, they obtained a function similar to that observed in three conditions of the present study (Panels 2, 3 , and 4 of Figure 1).

Either below-chance prediction success (i.e., 30\% correct) or an external locus of control was sufficient to lessen the correspondence between verbal expectancy and choice RT. Thus, when either of these factors was in effect, choice RT to incorrectly predicted stimuli did not reflect a direct relationship between prediction confidence and stimulus expectancy. That is, for these conditions, choice RT did not reflect a decrease in expectancy (or preparedness) for a nonpredicted stimulus when subjects showed increased prediction confidence for the nonpresented stimulus. Furthermore, when both of the above conditions were in effect (i.e., when externals predicted correctly on $30 \%$ of the trials), choice RT was not only uninfluenced by prediction confidence, but was not even significantly faster to predicted than to nonpredicted stimuli.

Every prior RT study that manipulated the frequency of subjects' correct stimulus predictions used random (rather than selected) volunteers from introductory psychology classes and found reliably faster reactions to correctly predicted than to incorrectly predicted stimuli when the probability of a correct prediction was only .30 (Geller, 1974; Whitman \& Geller, 1972, 1973). Thus, an external locus of control and an incompetent task competency were additive in increasing independence among three indexes of expectancy: a stimulus prediction, confidence in a prediction, and choice RT.

\section{REFERENCES}

Albert, M., \& Gelle r, E. S. Perceived control as a mediator of learned helplessness. American Journal of Psychology, 1978, 91, 389-400.

Gelle R, E. S. Preceding prediction outcome and prediction outcome probability: Interacting determinants of choice reaction time. Journal of Experimental Psychology, 1974, 103, 426-430.

Geller, E. S., \& Whitman, C. P. Confidence in stimulus predictions and choice reaction time. Memory \& Cognition, 1973, 1, 361-368.

Hiroto, D. S. Locus of control and learned helplessness. Journal of Experimental Psychology, 1974, 102, 187-193.

LEFCOURT, H. M. Locus of control: Current trends in theory and research. Hillsdale, N.J: Erlbaum, 1976.

Phares, E. J. Locus of control in personality. Morristown, N.J: General Learning Press, 1976.

RotTE R, J. B. Social learning and clinical psychology. Englewood Cliffs, N.J: Prentice-Hall, 1954.

Rotter, J. B. Generalized expectancies for internal versus external control of reinforcement. Psychological Monographs, 1966, 80(Whole No. 609).

Whitman, C. P., \& Geller, E. S. Manipulation and reversal of the probability of a correct stimulus prediction in a choice reaction task. Psychonomic Science, 1972, 29, 339-341.

Whitman, C. P., \& Geller, E. S. Prediction outcome distributions as determinants of choice reaction time. Perception \& Psychophysics, 1973, 13, 105-107.

(Manuscript received for publication April 21, 1983.) 